\title{
A Machine Vision Detection of Unauthorized On-Street Roadside Parking in Restricted Zone: An Experimental Simulated Barangay-Environment
}

\author{
Alvin Sarraga Alon ${ }^{1}$, Jesusimo L. Dioses, Jr. ${ }^{2}$ \\ ${ }^{1}$ Technological Institute of the Philippines, Manila Philippines, aalon.cpe@tip.edu.ph \\ ${ }^{2}$ Isabela State University, Echague Isabela Philippines, jdiosesjr@gmail.com
}

\begin{abstract}
The study developed a cost-effective framework for unauthorized parking detection using a machine-vision based deep learning method. The system was introduced on a Raspberry Pi 4b using the MobileNet SSD algorithm to detect vehicles illegally parked based on the live feed received from a Pi camera. The system was introduced to monitor unauthorized parking on a specific barangay simulated-roadside-parking lot. Results of the assessment indicate that the study was capable of identifying illegally parked vehicles with an overall performance rate of $96.16 \%$ and $98.93 \%$ respectively for legally and illegally parked vehicles, with a combined test resulting in $97.56 \%$. The study showed that the detection was robust to changes in light intensity and the presence of shadow effects in varying environmental conditions, due to the deep learning strength.
\end{abstract}

Key words: car zoning detection, illegal parking, machine vision, object detection.

\section{INTRODUCTION}

Illegal parking is a major urban challenge attributed to the increasingly growing amount of private cars and the rising demand for parking, as compared to the lack of parking resources [1]. In circumstances where drivers do not find a vacant parking spot after extended cruising around the parking lot, they can opt to park illegally [2]. Illegally parked vehicles not only cause car drivers inconvenience but also worsen the road congestion issue during peak hours because they appear to obstruct road traffic [3]. Regrettably, traditional security devices also depend primarily on human operators to patrol the parking lots in shifts during the day to locate automobiles illegally parked, which is ineffective for the continuous monitoring of unauthorized parking events [4]. Therefore, more drivers will seek their luck to park illegally and gamble on the likelihood that they would avoid punishment if they manage to flee before law enforcement personnel arrive [5], [6]. As such, an illegal parking monitoring device is required which can automate the task of detecting illegally parked vehicles in real-time.
In the study of [7], a methodology has been used to identify unauthorized parking in real time by introducing a modern image projection that decreases data dimensionality and hence decreases the computational difficulty of the segmentation and monitoring processes. The study inverts the transition after event detection to recover the vehicle's original appearance and to enable more processing which may involve 2-D information. The study measured algorithm performance using the i-LIDS vehicle detection challenge dataset as well as videos. Such videos evaluate the algorithm in a number of environmental conditions like video at night and instances of abrupt weather changes.

Another study for detection and identification of illegally parked vehicles was conducted by [8]. This paper presented a basic solution to image processing to detect and classify improperly parked vehicles. This study suggested the application of image processing algorithms to identify, extract, locate, segment and recognize vehicle number plates from the image acquired. This framework provided the concerned authority with near-real-time details of illegally parking automobiles through the message.

In this study of [9], proposed a framework for automatic detection of illegally parked vehicle. The system consisted of two main elements, namely object recognition and monitoring of movements. The study implemented one of the most prevalent YOLO (v3) object detection algorithms to identify vehicles and template matching methods using normalized cross correlation for monitoring movement. Experiments demonstrate that the approach presented can achieve very high precision and is resilient to various camera positions, environmental conditions and video illuminations.

Another deep learning was used by [10]. The study proposed a completely integrated system to perform illegal monitoring of parking from end to end. A deep learning-based object recognition algorithm, You Only Look Once Version 3 (YOLOv3), achieves robust and efficient identification of vehicles. Using template matching and Intersection over Union (IoU) measurements, movement tracking was used to measure the vehicle-in-violation stationary period with built-in error tolerance mechanisms. 
The objective of the study is to detect a car that is illegally parked on the roadside. Considering the different deep learning algorithm in detecting the objects [11]-[15]. A zoning method of detecting an illegal car is proposed to differentiate it from the boundary lanes of legal parking on the road. Also aiming for a system that will give barangay officials an automated detection of illegally parked cars within their vicinity. The increasing amount of cars parked on the roadsides which are mostly wrongly parked and not following the "No Parking" sign, built the possible use of this scheme.

The study will be used by the Barangay officials to track where and who the people who disobey the law are. Each section involves the other aspects of the system that relate to the question or issue that the study needs to investigate, as well as the limitations that have an impact on the study's ability to effectively explore the nature of the system. The scope and limitations of the study are to identify cars that are illegally parked and to warn the barangay office so that the officials know where the car is. Trees, waiting sheds restrict the detection field. Partial obstruction (with a tree canopy) functions in some situations and not in others. Similarly, several of the cars inside the camera's lot would be concealed behind several in front of the camera. This process, however, is limited by small images, odd viewing angles, visibility, and obstruction. There's no way you can put a camera above the floor in most of the basement parking lots. Trees, Pills, Posts, etc. can block the field of view in certain places.

\section{METHODOLOGY}

The study's basic goal is to detect the cars that are illegally parked on the roadside. Implementing the core requirements given, The study was able to construct a block diagram as shown in Figure 1.

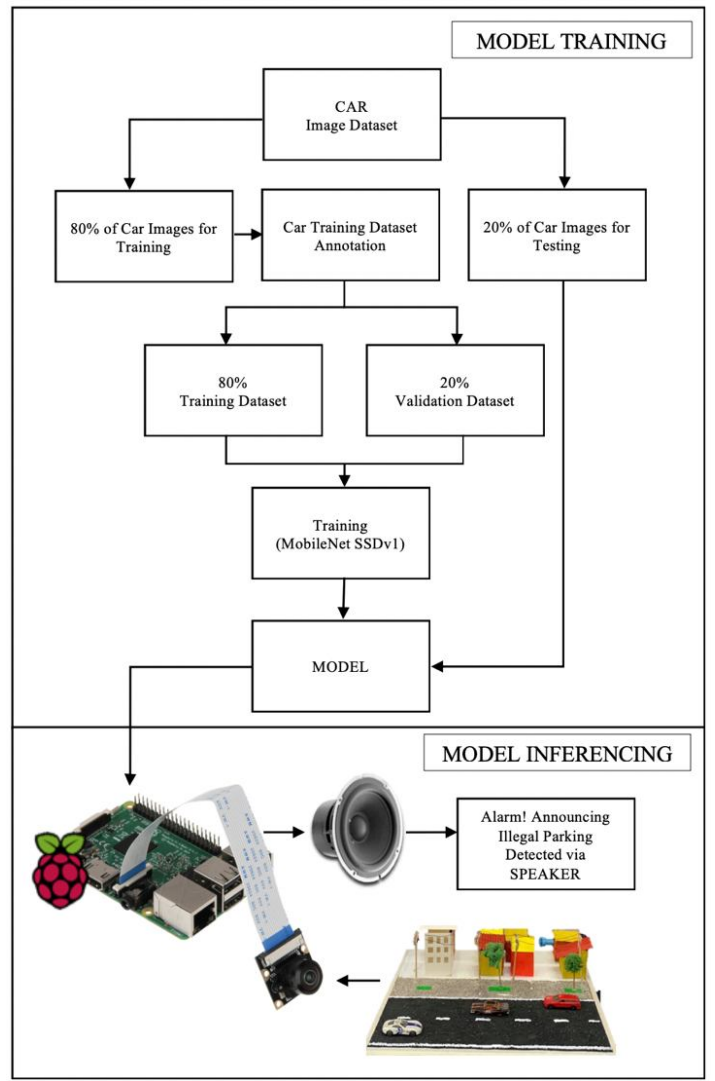

Figure 1: Methodology of Road-Side Illegal Parking

\subsection{Car Dataset (Matchbox Cars)}

The dataset composed of gathered images through video to frame image conversion as shown in Figure 2. Matchbox cars were used within the dataset for better detection. Throughout, the dataset reached 2348 images.

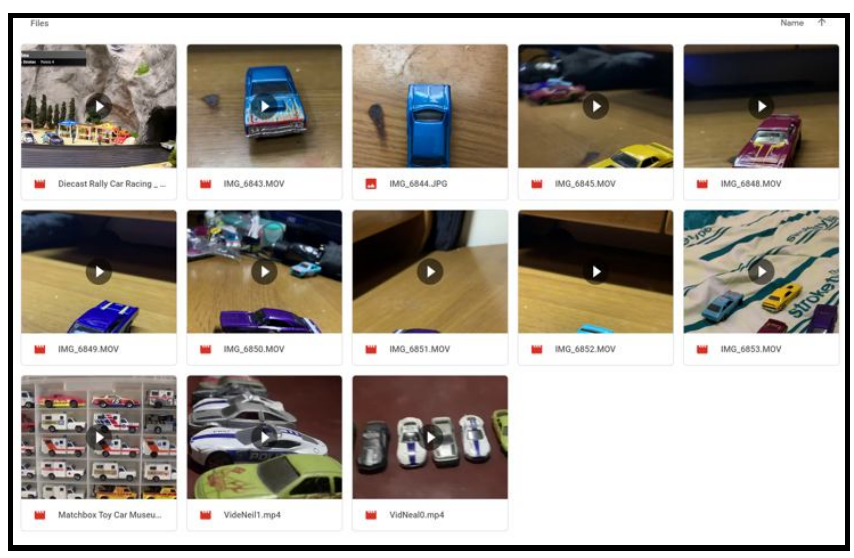

Figure 2: Videos Gathered for Dataset

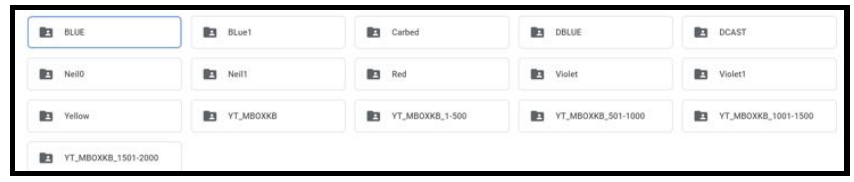

Figure 3: Converted Images from the Video Files 


\subsection{Resize Image}

The images were resized, each image should be less than $200 \mathrm{~kb}$ and resolution shouldn't be more than $720 \times 1280$ as shown in Figure 3.

\subsection{Splitting of Images}

The gathered dataset was split $20 \%$ for test and $80 \%$ for the train as shown in Figure 4 and Figure 5. This was based on the best practice done by the majority. Also, deletion of not needed images has been done during this process.

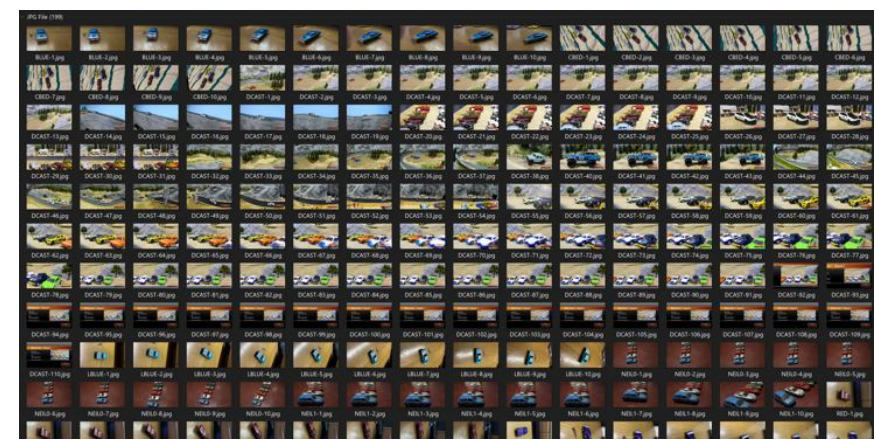

Figure 4: Images within Test Folder

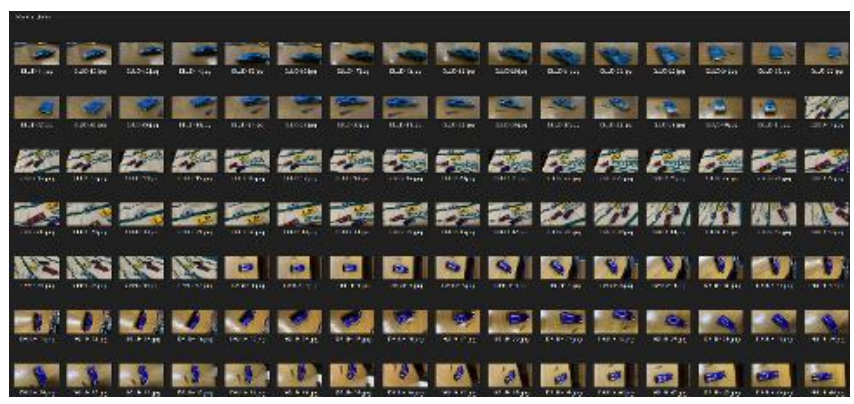

Figure 5: Images within Train Folder

\subsection{Images Annotation}

Each image gathered has been annotated using labellmg as shown in Figure 6. The label used for every image was "car". The output was an XML file containing the coordinates of the car.

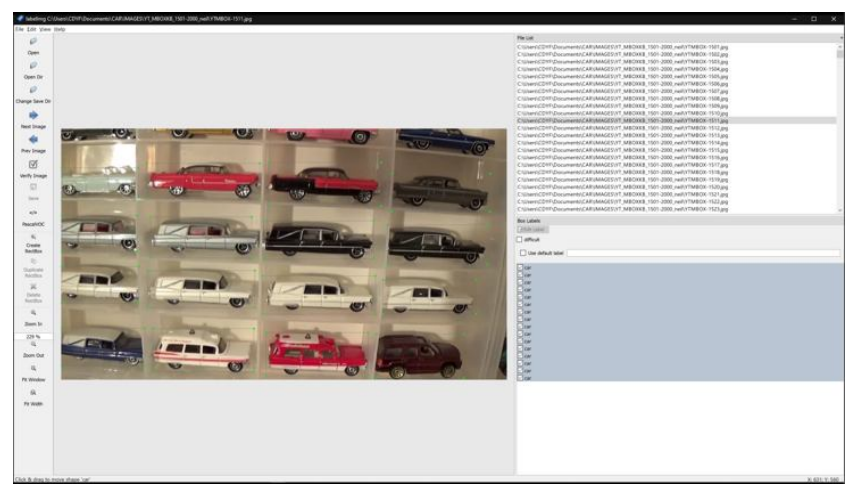

Figure 6: LabelImg Sample Window

\subsection{Training using SSD MobileNet}

The study used a pre-trained model from the TensorFlow model zoo. The training was continued until the loss reaches less than 2 as shown in Figure 7.

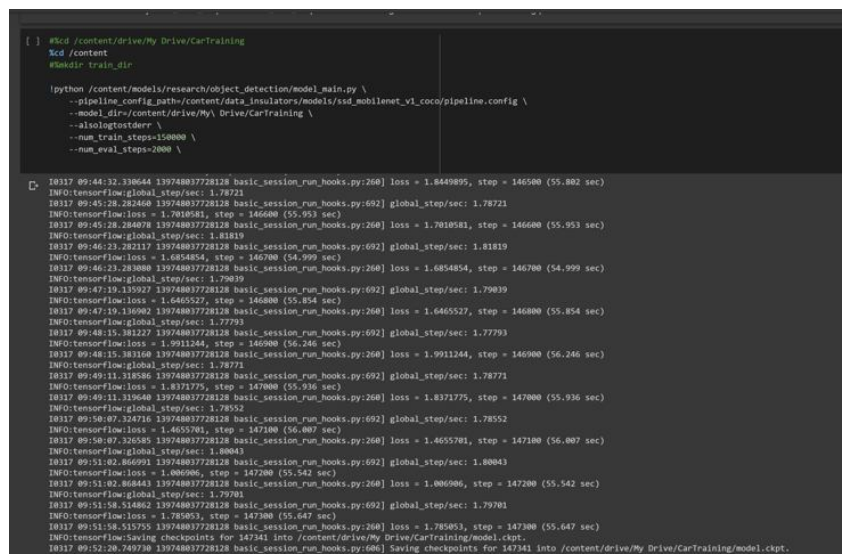

Figure 7: Training using Google Colab

\subsection{Model Generation}

The output files of training are shown in Figure 8, to use this file, the study needed to note the highest model number and use inference_graph.py to generate a protobuf file has been used for detecting the object as shown in Figure 9.

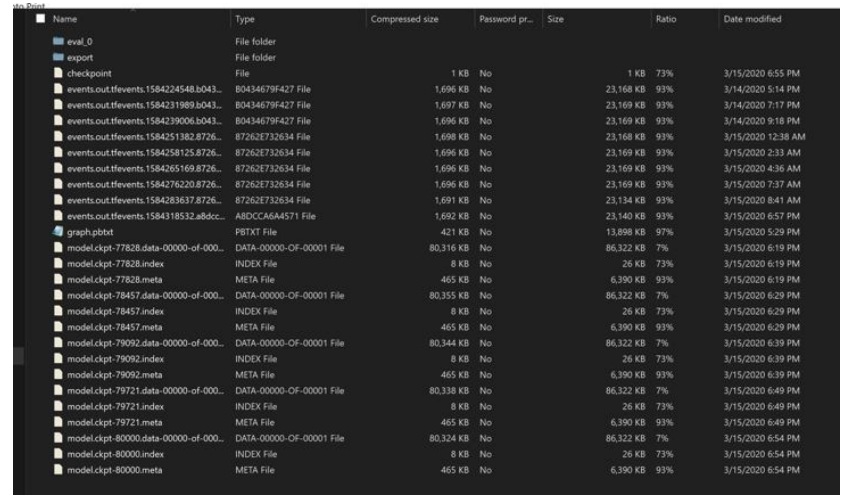

Figure 8: Files after Training

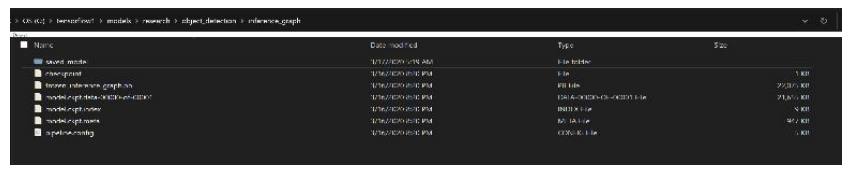

Figure 9: Files after running inference_graph.py

\subsection{Car Detection using Zoning}

Once the model is done and ready to use, the file has been put to raspberry and using the Pi camera as the way for detecting the car. The study has now been implemented to the core system to form zoning within the area where the Pi camera is angled. 


\section{HARDWARE DESIGN}

Figure 10 shows the miniature prototype barangay environment to where the system can simulate the parking lane and not parking lane of the roads. Thus, giving the flexibility of what the different scenarios or angles where Pi camera should be facing when deployed.

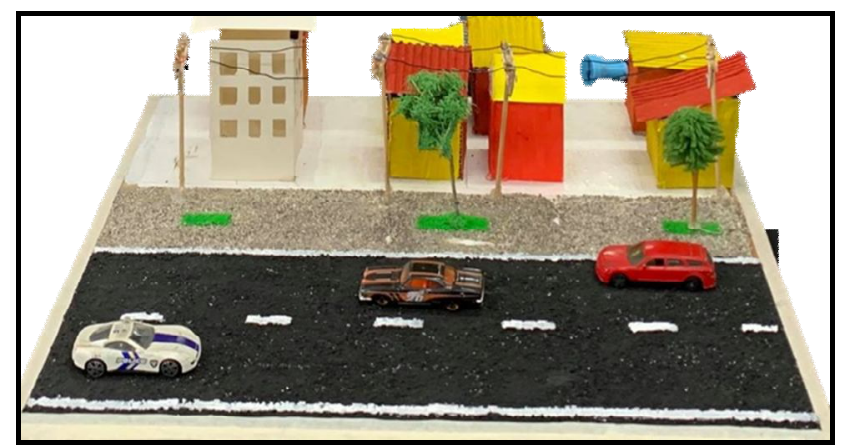

Figure 10: Prototype of the Simulated Barangay-Environment

\section{RESULTS AND DISCUSSIONS}

\subsection{Training Results}

Mean average precision measures how accurate the predictions are, it is the percentage of correct detections. Figure 11 and 12 shows the mean average precisions at different IoU.

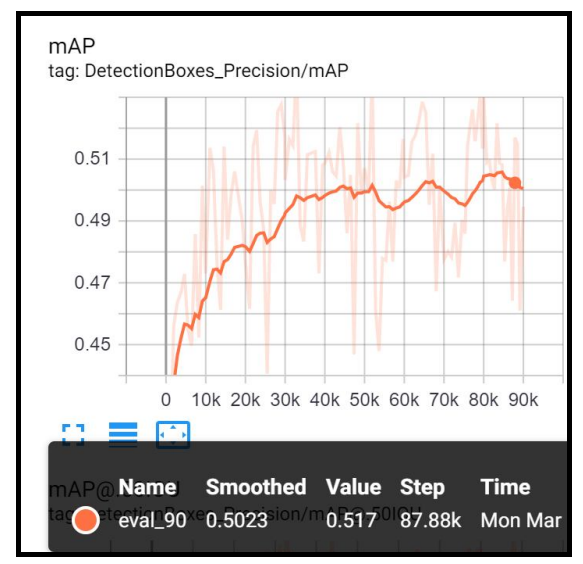

Figure 11: Mean Average Precision

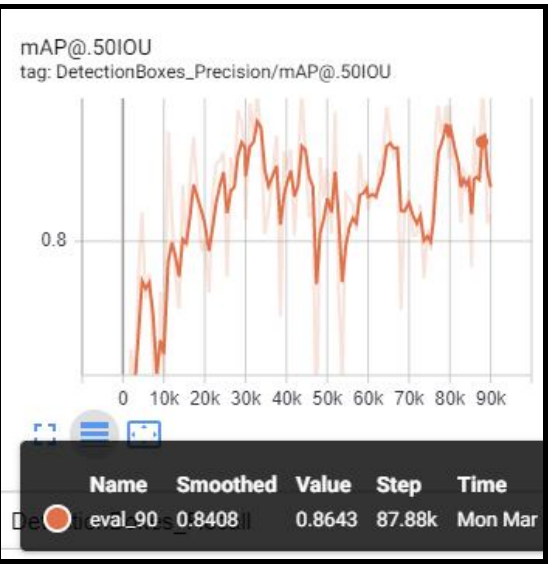

Figure 12: Mean Average Precision @ 50\% IOU

Mean average recall measures how accurate the model finds all the positives. Figure 13 and 14 shows the average recall values at different sizes.

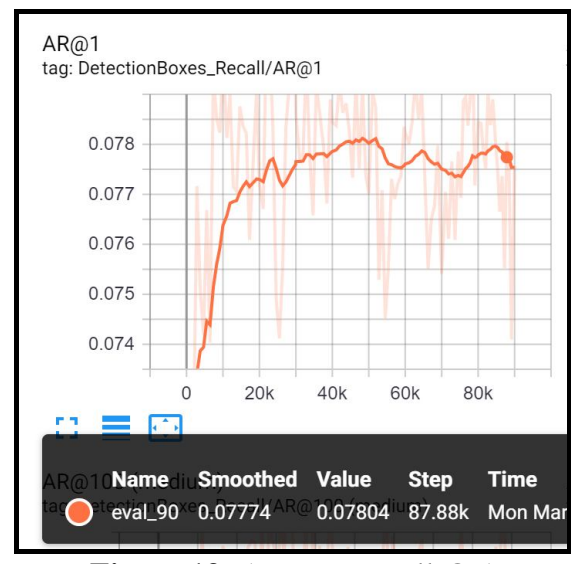

Figure 13: Average Recall @ 1

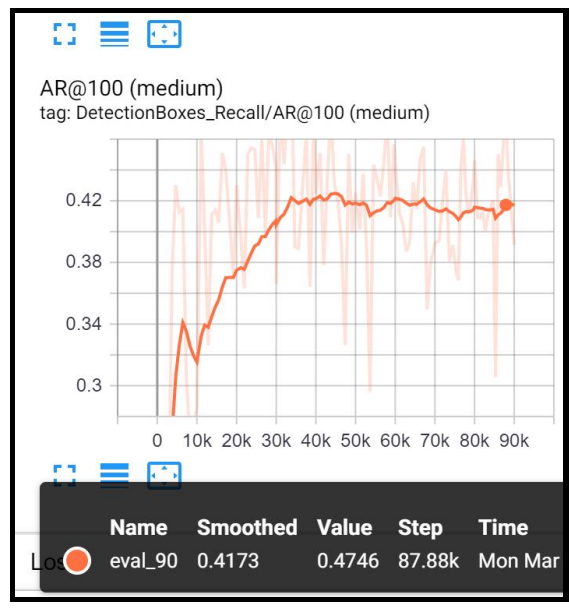

Figure 14: Average Recall @ 100

IoU tests the boundary difference between two objects. The analysis used to calculate how far the predicted boundary overlaps with ground truth (the true boundary of objects). The study predefines an IoU level (say 0.5) in certain datasets while classifying whether the forecast is a true positive or a false positive. 


\subsection{Testing Results}

Figure 15 shows the testing results of the illegal parking of cars using zoning. The dataset composed of gathered test images from video to frame image conversion.

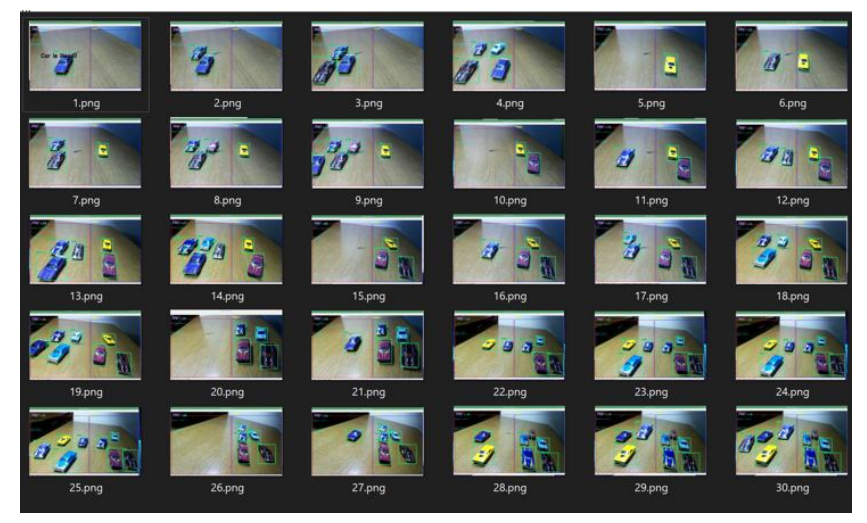

Figure 15: Testing Results

Table 1 shows the actual overall testing using the images from Figure 15. It shows a total of 30 test cases of legally and illegally parked cars on the road-side.

Table 1: Actual Testing Results

\begin{tabular}{|c|c|c|c|c|c|c|}
\hline \multirow[t]{2}{*}{$\begin{array}{l}\text { Test } \\
\text { No. }\end{array}$} & \multicolumn{2}{|c|}{ Test Case } & \multicolumn{2}{|c|}{ Expected Output } & \multicolumn{2}{|c|}{$\begin{array}{c}\text { Actual Output } \\
\text { (\%Detection) }\end{array}$} \\
\hline & Legal & Illegal & Legal & Illegal & Legal & Illegal \\
\hline 1 & 0 & 0 & - & - & - & - \\
\hline 2 & 0 & 1 & - & $\checkmark$ & - & $99 \%$ \\
\hline 3 & 0 & 2 & - & $\checkmark$ & - & $99 \%$ \\
\hline 4 & 0 & 3 & - & $\boldsymbol{V}$ & - & $99 \%$ \\
\hline 5 & 0 & 4 & - & $\checkmark$ & - & $99 \%$ \\
\hline 6 & 1 & 0 & $\sqrt{ }$ & - & $99 \%$ & - \\
\hline 7 & 1 & 1 & $\checkmark$ & $\checkmark$ & $99 \%$ & $99 \%$ \\
\hline 8 & 1 & 2 & $\sqrt{ }$ & $\boldsymbol{V}$ & $99 \%$ & $99 \%$ \\
\hline 9 & 1 & 3 & $\checkmark$ & $\checkmark$ & $99 \%$ & $99 \%$ \\
\hline 10 & 1 & 4 & $\sqrt{ }$ & $\checkmark$ & $99 \%$ & $99 \%$ \\
\hline 11 & 2 & 0 & $\checkmark$ & - & $99 \%$ & - \\
\hline 12 & 2 & 1 & $\sqrt{ }$ & $\checkmark$ & $99 \%$ & $99 \%$ \\
\hline 13 & 2 & 2 & $\sqrt{ }$ & $\checkmark$ & $99 \%$ & $99 \%$ \\
\hline 14 & 2 & 3 & $\sqrt{ }$ & $\checkmark$ & $99 \%$ & $99 \%$ \\
\hline 15 & 2 & 4 & $\checkmark$ & $\checkmark$ & $99 \%$ & $99 \%$ \\
\hline 16 & 3 & 0 & $\sqrt{ }$ & - & $99 \%$ & - \\
\hline 17 & 3 & 1 & $\checkmark$ & $\checkmark$ & $99 \%$ & $99 \%$ \\
\hline 18 & 3 & 2 & $\sqrt{ }$ & $\checkmark$ & $99 \%$ & $99 \%$ \\
\hline 19 & 3 & 3 & $\sqrt{ }$ & $\checkmark$ & $99 \%$ & $99 \%$ \\
\hline 20 & 3 & 4 & $\checkmark$ & $\checkmark$ & $99 \%$ & $99 \%$ \\
\hline 21 & 4 & 0 & $\sqrt{ }$ & - & $97.25 \%$ & - \\
\hline 22 & 4 & 1 & $\checkmark$ & $\checkmark$ & $97 \%$ & $99 \%$ \\
\hline 23 & 4 & 2 & $\sqrt{ }$ & $\sqrt{ }$ & $99 \%$ & $98.5 \%$ \\
\hline 24 & 4 & 3 & $\sqrt{ }$ & $\checkmark$ & $99 \%$ & $98.5 \%$ \\
\hline 25 & 4 & 4 & $\sqrt{ }$ & $\checkmark$ & $99 \%$ & $98.5 \%$ \\
\hline 26 & 5 & 0 & $\sqrt{ }$ & - & $97.6 \%$ & - \\
\hline 27 & 5 & 1 & $\sqrt{ }$ & $\checkmark$ & $98.6 \%$ & $99 \%$ \\
\hline 28 & 5 & 2 & $\checkmark$ & $\checkmark$ & $77.4 \%$ & $99 \%$ \\
\hline 29 & 5 & 3 & $\sqrt{ }$ & $\checkmark$ & $79 \%$ & $99 \%$ \\
\hline \multirow[t]{2}{*}{30} & 5 & 4 & $\sqrt{ }$ & $\checkmark$ & $75 \%$ & $99 \%$ \\
\hline & & & $\mathrm{Mec}$ & Average & $96.16 \%$ & $98.93 \%$ \\
\hline
\end{tabular}

\section{CONCLUSION AND FUTURE WORKS}

In this study, the system is an economical outdoor illegal parking detection system that has been implemented using MobileNet SSD at a particular barangay-environment. The study overcame the problem of illegal parking by being able to automatically extract image features and display robustness against road-side parking. Excellent detection was obtained when testing the device at a distinct time of a day under various light and shadow patterns. The system gathered a testing accuracy for legally and illegally parked vehicles of $96.16 \%$ and $98.93 \%$ respectively.

Using the system, the number of illegal parking incidents may be substantially decreased because it imitates the presence of enforcement officers to hold such offenders away, thus lightening the load on enforcement officers and allowing a more robust system of illegal parking enforcement. Incorporating the system with current smart parking solutions will also boost parking conditions for drivers because they would travel around the parking lot easily without interruption. In the future, the research image collection will be expanded by adding additional images of cars in varying colors and sizes to further enhance the quality of unauthorized parking detections.

\section{ACKNOWLEDGEMENT}

The author would like to thank particularly his Microprocessor's Class of 2nd SEM SY19-20. Additionally, to MR-SUAVE Lab, and Microprocessors Lab of the Technological Institute of the Philippines.

\section{REFERENCES}

1. C. Morillo and J. M. Campos, On-street Illegal Parking Costs in Urban Areas, Procedia - Social and Behavioral Sciences, vol. 160, pp. 342-351, 2014. https://doi.org/10.1016/j.sbspro.2014.12.146

2. T. T. M. Thanh and H. Friedrich, Legalizing the illegal parking, a solution for parking scarcity in developing countries, Transportation Research Procedia, vol. 25, pp. 4950-4965, 2017. https://doi.org/10.1016/j.trpro.2017.05.374

3. C. Spiliopoulou and C. Antoniou, Analysis of Illegal Parking Behavior in Greece, Procedia - Social and Behavioral Sciences, vol. 48, pp. 1622-1631, 2012.

4. J. Parmar, P. Das, and S. M. Dave, Study on demand and characteristics of parking system in urban areas: A review, Journal of Traffic and Transportation Engineering (English Edition), vol. 7, no. 1, pp. 111-124, 2020. https://doi.org/10.1016/j.jtte.2019.09.003

5. X. Ma, X. Sun, Y. He, and Y. Chen, Parking Choice Behavior Investigation: A Case Study at Beijing Lama Temple, Procedia - Social and Behavioral Sciences, vol. 96, pp. 2635-2642, 2013. 
6. Á. Costa, C. Rocha, and S. Melo, Parking Management Policies and the Effectiveness of Public Policy Solutions, Procedia - Social and Behavioral Sciences, vol. 111, pp. 965-973, 2014.

https://doi.org/10.1016/j.sbspro.2014.01.131

7. J. T. Lee, M. S. Ryoo, M. Riley and J. K. Aggarwal, Real-Time Illegal Parking Detection in Outdoor Environments Using 1-D Transformation, in IEEE Transactions on Circuits and Systems for Video Technology, vol. 19, no. 7, pp. 1014-1024, July 2009.

8. S. G. Kashid and S. A. Pardeshi, Detection and identification of illegally parked vehicles at no parking area, 2014 International Conference on Communication and Signal Processing, Melmaruvathur, 2014, pp. 1025-1029. https://doi.org/10.1109/ICCSP.2014.6950002

9. W. Chen and C. K. Yeo, Unauthorized Parking Detection using Deep Networks at Real Time, 2019 IEEE International Conference on Smart Computing (SMARTCOMP), Washington, DC, USA, 2019, pp. 459-463.

10. Z. Liu, W. Chen and C. K. Yeo, Automatic Detection of Parking Violation and Capture of License Plate, 2019 IEEE 10th Annual Information Technology, Electronics and Mobile Communication Conference (IEMCON), Vancouver, BC, Canada, 2019, pp. 0495-0500.

11. Z. Chen, T. Ellis and S. A. Velastin, Vehicle detection, tracking and classification in urban traffic, 2012 15th International IEEE Conference on Intelligent Transportation Systems, Anchorage, AK, 2012, pp. 951-956. https://doi.org/10.1109/ITSC.2012.6338852

12. A. S. Alon, A YOLOv3 Inference Approach for Student Attendance Face Recognition System, International Journal of Emerging Trends in Engineering Research, vol. 8, no. 2, pp. 384-390, 2020. https://doi.org/10.30534/ijeter/2020/24822020

13. F. J. P. Montalbo and A. A. Hernandez, Classification of Fish Species with Augmented Data using Deep Convolutional Neural Network, 2019 IEEE 9th International Conference on System Engineering and Technology (ICSET), 2019. https://doi.org/10.1109/ICSEngT.2019.8906433

14. N. K. Gattim, Plant Leaf Disease Detection Using SVM Technique, International Journal of Emerging Trends in Engineering Research, vol. 7, no. 11, pp. 634-637, 2019. https://doi.org/10.30534/ijeter/2019/367112019

15. N. S. C, Heuristics of Machine Learning on Embedded Intelligence Device for Multiple Object Detection, International Journal of Emerging Trends in Engineering Research, vol. 8, no. 3, pp. 787-791, 2020. https://doi.org/10.30534/ijeter/2020/29832020 